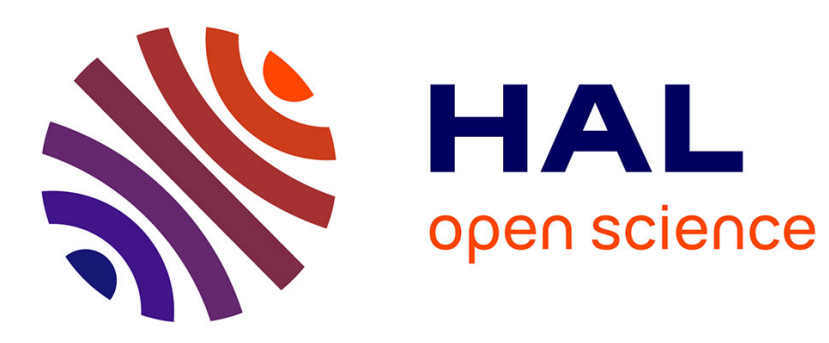

\title{
UDP PING: a dedicated tool for improving measurements of the Internet topology
}

Fabien Tarissan, Elie Rotenberg, Matthieu Latapy, Christophe Crespelle

\section{To cite this version:}

Fabien Tarissan, Elie Rotenberg, Matthieu Latapy, Christophe Crespelle. UDP PING: a dedicated tool for improving measurements of the Internet topology. MASCOTS'14: IEEE 22nd International Symposium on Modeling Analysis and Simulation of Computer and Telecomunication Systems, Sep 2014, Paris, France. pp.506 - 509, 10.1109/MASCOTS.2014.74 . hal-01208361v2

\section{HAL Id: hal-01208361 \\ https://hal.science/hal-01208361v2}

Submitted on 19 Oct 2015

HAL is a multi-disciplinary open access archive for the deposit and dissemination of scientific research documents, whether they are published or not. The documents may come from teaching and research institutions in France or abroad, or from public or private research centers.
L'archive ouverte pluridisciplinaire HAL, est destinée au dépôt et à la diffusion de documents scientifiques de niveau recherche, publiés ou non, émanant des établissements d'enseignement et de recherche français ou étrangers, des laboratoires publics ou privés. 


\title{
UDP PING: a dedicated tool for improving measurements of the Internet topology
}

\author{
Fabien Tarissan*, Élie Rotenberg*†, Matthieu Latapy* and Christophe Crespelle ${ }^{\dagger}$ \\ *Sorbonne Universités, UPMC Université Paris 6 and CNRS, UMR 7606, LIP6, Paris \\ †Université Claude Bernard Lyon 1, DANTE/INRIA, LIP UMR CNRS 5668, ENS de Lyon, Université de Lyon
}

\begin{abstract}
The classical approach for Internet topology measurement consists in distributively collecting as much data as possible and merging it into one single piece of topology on which are conducted subsequent analysis. Although this approach may seem reasonable, in most cases network measurements performed in this way suffer from some or all of the following limitations: they give only partial views of the networks under concern, these views may be intrinsically biased, and they contain erroneous data due to the measurement tools. Here we present a new tool, named UDP PING, that relies on a very different approach for the measurement of the Internet topology. Its basic principle is to measure the interface of a given target directed toward a monitor which sends the measurement probe. We demonstrate how to use it to deploy real world-wide measurements that provide reliable (i.e. bias and error free) knowledge of the Internet topology, namely the degree distribution of routers in the core Internet in our example.
\end{abstract}

\section{INTRODUCTION}

It appeared a decade ago that the Internet topology has features which make it very different from classical assumptions and models. As these properties were highly counter-intuitive, they received much attention, and much effort has been devoted to understand them and capture them into relevant models. Nowadays, measuring and modeling Internet-like topologies has become a well-recognized area of research in itself. Indeed, it makes no doubt that features observed in Internet measurements should be part of our modeling effort. However, gaining accurate and reliable knowledge of actual properties of the Internet topology is challenging. As stated by W.Willinger (in [1], p.586), "A very general but largely ignored fact about Internet-related measurements is that what we can measure in an Internet-like environment is typically not the same as what we really want to (or what we think we actually measure)". Indeed, the Internet is a huge and complex system composed of many different (sometimes buggy) protocol implementations, heterogeneous and poorly documented devices, operators implementing different (and often not measurement-friendly) policies, etc. As a consequences, data obtained from measurements is partial, and often biased. Going further, understanding such data and deriving appropriate conclusions is a challenge in itself and it relies on a deep knowledge of the actual deployment of infrastructures, both nowadays and in the past as old devices/software/protocols are mixed with the most modern ones. As a consequence, current knowledge of Internet topology remains limited, as well as confidence in previously published studies.

The classical approach for Internet topology measurement consists in collecting as much data as possible (using for instance traceroute of Border Gateway Protocol (BGP) tables) and in constructing from it a view of the topology by merging the obtained data. Although this approach may seem reasonable, in most cases network measurements give partial views of the networks under concern, contain erroneous data for the reasons cited above, and may moreover be intrinsically biased. For instance, it is shown in [2], [3] (both experimentally and formally) that the degree distribution observed on measurements of the Internet topology may be significantly biased by the measurement procedure. Similar problems occur for other properties and other networks [4], [5], [6], [7]. This has crucial consequences for the field. For instance, the results claiming that the Internet is very resilient to failures but sensitive to attacks [8], [9], [10], [11] rely on the assumption that the Internet has a power-law degree distribution with a given exponent, which is observed in measurements [12]. The fact that such degree distributions may be observed even if the underlying network has a totally different degree distribution [2], [3]) makes the relevance of these results unclear. This leads to difficult discussions and analyses of the extent to which the observed degree distribution may be trusted [13], [4].

This problem is nowadays widely acknowledged: as network measurements rely on intricate procedures which give limited views of the network, the obtained views may have properties induced by the measurement procedure, and thus differ significantly from the ones of the studied network. However, only very limited and unsatisfactory solutions exist to cope with this problem: despite a few exceptions [5], [6], [7], most results on this topic are negative and show that the observed properties should not be trusted [2], [3], [5]. One approach could be to derive the properties of the network of interest from the observed ones (rather than simply consider that the observed ones are true), but this turns out to be very difficult. Another approach could be to conduct larger measurements, and this is indeed done (Caida [14], Dimes [15]). However, the network to measure generally evolves faster than our ability to measure it, and thus such approaches, even though they provide interesting data, do not solve the problem.

\section{OвJеCTIVE}

The degree distribution of a network topology (i.e. for each integer $k$ the fraction $p_{k}$ of nodes with $k$ links) is one of its most basic properties. It has a strong impact on 
key features of the network like efficiency of protocols and robustness to failures and attacks. Until the seminal papers [16], [12] in the late 90's, it was commonly assumed that the degree distribution of the Internet followed a homogeneous law, generally modeled by a Poisson distribution. Based on actual measurements, though, these papers gave evidences of the fact that it may be much more heterogeneous, better modeled by a power-law. Since then, much effort is devoted to studying this distribution and capturing it in appropriate models of the Internet.

However, as stated above, recent works have shown that the observed degree distribution of the Internet may be significantly biased by the measurement process. Indeed, it is deduced from partial maps obtained through intricate and unreliable measurements which tend to give biased views.

Our objective here is to show the implementation of a new approach able to rigorously estimate the degree distribution of the Internet, in a much more reliable way than before. Instead of building maps of this topology, we design a method to accurately estimate the degree of a given router; then we build a process that allows to select core routers uniformly at random, and estimate their degree. We therefore obtain an accurate estimate of the degree of a set of random nodes in the considered topology, representative of the whole.

It is worth noticing that the present work is related to two complementary papers. In [17], we showed by simulations that the approach succeeds in accurately estimating the degree distribution of various topologies and is free from bias. In [18], we deployed a real world-wide measurement campaign using this tool and we presented the first results related to the degree distribution of routers in the core Internet.

\section{PRINCIPLES}

Our approach relies on our ability to accurately measure the degree of a given core router and to uniformly sample core routers (independently from their degree). We then measure the degree of such random routers and obtain the degree distribution of a representative set of routers. If the set is large enough, this degree distribution is close to the one of the whole network.

\section{A. Measuring the degree of a given router}

When a machine in the Internet receives an invalid packet from a sender $S$, it is in general supposed to answer to $S$ with an error message using the dedicated ICMP protocol. Many tools, including traceroute and many alias resolution tools, rely on this feature to generate errors which provide information regarding the network. An important feature of such error messages is that they are in principle sent using the interface of the machine that routes packets towards $S$. Therefore, by generating an error on a target $t$, a monitor $m$ obtains an interface of $t$. If many monitors distributed in the Internet do so, then they will probably obtain all interfaces of the target, and so its degree.
We implement here this approach with UDP PING, a tool that we designed and implemented, which sends a UDP packet to a target on an unallocated port. See Section IV-A for details on the implementation and behavior of this tool.

Notice that not all routers answer to such probes, and that some routers always use the same interface to answer. Likewise, some routers may answer with random interfaces. We will handle such issue below. The key point here is that, given an appropriate set of monitors and a correct target, we are able to accurately estimate its degree. This is already an improvement over previous situation, where no such tool existed and where the degree of a node could only be guessed from maps obtained with traceroute-based measurements.

\section{B. Sampling targets}

With the ability to reliably estimate the degree of a single router as explained above, one may estimate the degree distribution of a set of nodes (by applying the method to each node in the set). If this set is a set of nodes taken uniformly at random in the core Internet (i.e. all the nodes have the same probability to be chosen) then the obtained degree distribution is itself an approximation of the degree distribution of the core Internet, and the larger the sample, the better the approximation. However, choosing uniformly at random a node in the core Internet is not possible in general. We explain here how to bypass this issue.

First notice that it is easy to sample a random IP address, as this is noting but a 32 bit integer. But a random IP address is not a random node, as routers may have several interfaces (aka IP addresses) and then the probability to sample a router by sampling a random address is proportional to its number of interfaces. As a consequence, if we know the degree distribution $\left(p_{k}\right)$ of nodes corresponding to IP addresses sampled uniformly at random, then the degree distribution of the set of all nodes is nothing but $\left(p_{k} / k\right)$.

In summary, although we are unable to sample uniformly at random nodes in the core Internet, we are able to sample uniformly at random IP addresses and infer from the degree distribution of the corresponding nodes the one of the whole core Internet.

In addition, the random IP addresses we sample may belong to routers outside the core, to end-hosts, or even to routers in the core behaving incorrectly (they do not answer to probes or always use the same interface to answer). We easily identify IP addresses which do not belong to core routers: we see only one interface for them during our measurements, and simply discard them (as a consequence, we obtain no estimate of $p_{1}$, the fraction of nodes of degree 1 ). Notice that core routers which behave incorrectly also lead to nodes observed with degree 1 , or even 0 , and discarding them is correct. This induces no bias on the observed degree distribution if their behavior is not correlated to their degree, which seems a reasonable assumption. 


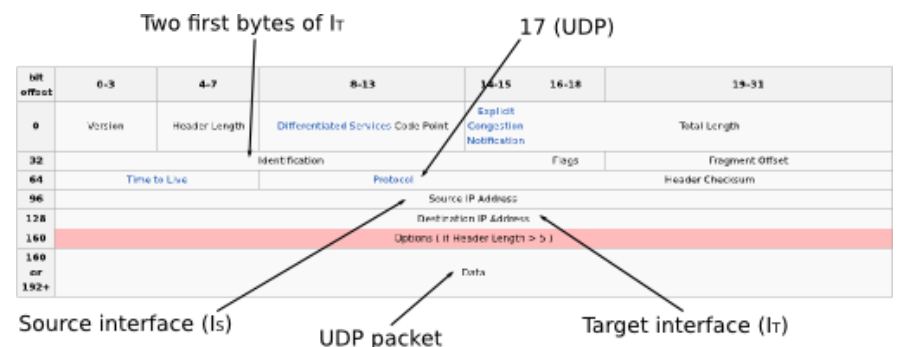

Fig. 1. UDP PING: IP Packet structure

Finally, we obtain a practical method to sample a set of uniformly random IP addresses of core routers, and to infer from their degree distribution the one of the whole core Internet. The quality of this estimate obviously depends on the quality of our set of monitors and on the size of our set of IP addresses. In-depth study of the achieved quality is a challenging task which belongs to future work, but we will discuss below ways to do so.

\section{DESCRIPTION OF THE TOOL}

The implementation of the approach presented above led to the design of UDP PING, available at http://www.rotenberg. io/udpping/. We present below details of the implematation and how to use it to deploy a coordinated and distributed measurement campaing.

\section{A. UDP PING}

UDP PING is a measurement tool developed to discover interfaces of machines in the Internet. It is run on a machine $\mathrm{M}$ called the monitor. It takes the following parameters:

- $\quad$ Source interface $\left(I_{S}\right)$. An 4-byte integer corresponding to an interface (IP address) of $\mathrm{M}$ on which UDP PING is executed. When $M$ is an end-host, it often has only one interface, called the IP address of M.

- Target interface $\left(I_{T}\right)$. A 4-byte integer corresponding to an IP address.

- UDP Destination Port (P). A 2-byte integer in the 49152 - 65535 range. UDP Ports in this range are assumed to be usually unallocated.

The goal of UDP PING is to detect if there is an active host $\mathrm{T}$ (the target) that owns the address $I_{T}$ and, if such T exists, to obtain an interface $I_{T^{\prime}}$ of $\mathrm{T}$ that is used by T to send packets towards $I_{S}$ (belonging to $\mathrm{M}$ ). To do so, UDP PING forges an IP packet (see Fig. 1), carrying an UDP packet (see Fig. 2). The destination address of the IP packet is $I_{T}$. The destination port of the UDP packet is P. $I_{T}$ is split in two 2-byte parts. The first 2-byte part is stored in the ID row of the IP packet header. The second 2-byte part is stored in the UDP Source Port row of the UDP message. By doing so, all the 4 bytes of $I_{T}$ are stored in the packet/message headers. The data field of the UDP packet contains a signature (typically a contact e-mail address) to allow network administrators to contact the sender upon receiving the message.

The IP/UDP packet is then sent by UDP PING through $I_{S}$. Once the packet is sent, UDP PING listens to all incoming

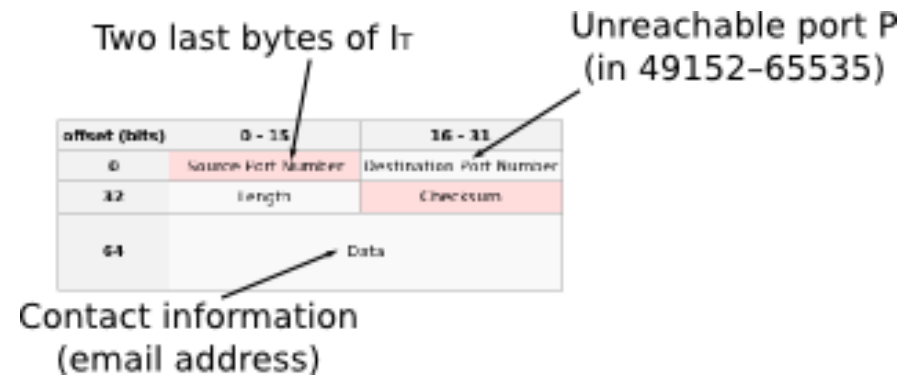

Fig. 2. UDP PING: UDP Packet structure

ICMP messages. If $I_{T}$ corresponds to an active host $\mathrm{T}$, then after receiving the packet, it detects that $\mathrm{P}$ is unreachable. $\mathrm{T}$ generates a type 3 ICMP message (Destination Unreachable), with error code 3 (Port Unreachable). This ICMP message also contains the headers of the incriminating packet, and in particular, the original IP ID row and UDP Source Port row. T then chooses one of its interfaces, $I_{T^{\prime}}$, according to its routing policy, to send the ICMP message back to its sender, $I_{S}$. Note that if $\mathrm{T}$ has more than one interface, $I_{T^{\prime}}$ can be different from $I_{T}$. Eventually, UDP PING catches this ICMP message. It extracts the following information:

- ICMP attached information, including the original IP ID row and UDP Source Port row. This allows UDP PING to reconstruct the 4-byte integer corresponding to $I_{T}$, and to identify this ICMP message as the expected one.

- $\quad$ Source address of the ICMP message - that is $I_{T^{\prime}}$.

UDP PING then exits with a success code and returns $I_{T^{\prime}}$. After a set amount of time, if UDP PING has not caught any ICMP message properly identified as the expected one (using the 4-byte integer reconstruction), it exits with a failure code. This can happen for multiple reasons, the most common being:

- $I_{T}$ does not belong to any connected machine (target offline)

- $\quad I_{T}$ does belong to an existing, active machine $\mathrm{T}$, but $\mathrm{T}$ discards UDP errors without sending ICMP error messages back

- $\quad I_{T}$ correspond to an active machine $\mathrm{T}$ that generates an ICMP error message but this message is filtered on its way back to $M$ (usually near $\mathrm{T}$ ).

- $\quad I_{T}$ is located beyond a firewall that silently discards unsolicited UDP traffic.

- $\quad \mathrm{P}$ is used by $\mathrm{T}$, making it effectively reachable (and then no error is generated at the network level).

\section{B. Deploying UDP PING}

Since $I_{T^{\prime}}$ can vary depending on $I_{S}$ (and therefore depending on M), UDP PING may be used from multiple monitors to get a list of distinct interfaces belonging to the same target. Using UDP PING from a set of monitors towards the same target $I_{T}$ is called Distributed UDP PING. If the set of monitors is large enough and well distributed in the Internet, one may then obtain several (and even all) interfaces of the target. 
Note that UDP PING requires privileges not only to execute binary code on the monitor, but also to forge and send packets at a very low level, and to listen and decode all the incoming ICMP messages. On most UNIX system, UDP PING therefore requires root privileges, which can be restrictive. However, if one disposes of a set of monitors and is granted such privileges, UDP PING can be effectively distributed using shell scripts and ssh tools to launch remote execution of the tool an retrieve the answers.

Since receiving a large amount of UDP messages from distributed machines can look like a distributed attack by the target host, or even unintentionally disable it, extreme care must be taken not to send all the UDP messages at once. A delay can (and should) be set between the sending of successive UDP PING probes from the different monitors. Likewise, all monitors should use the same target port, or else the measurement may look like a UDP port scanning. Once all these precautions are taken, the tool has no specific parameters to tune and is rather simple to use.

\section{CONCLUSION}

In the work, we presented the details of the implementation of a new method able to accurately estimate the degree distribution of routers in the core Internet. We showed the principle on which it relied, how it can be used to measure the degree of a single router and how it can be deployed for a coordinated distributed measurement campaign, leading to an estimationg of the degree distribution of such routers.

\section{ACKNOWLEDGMENT}

This work is partly supported by the European Commission EULER project (FP7 FIRE grant 258307) and by the Agence Nationale de la Recherche DynGraph grant ANR-10-JCJC0202 .

\section{REFERENCES}

[1] W. Willinger, D. Alderson, and J. Doyle, Mathematics and the Internet: A source of enormous confusion and great potential. Defense Technical Information Center, 2009.

[2] A. Lakhina, J. Byers, M. Crovella, and P. Xie, "Sampling biases in ip topology measurements," in INFOCOM 2003. Twenty-Second Annual Joint Conference of the IEEE Computer and Communications. IEEE Societies, vol. 1. IEEE, 2003, pp. 332-341.

[3] D. Achlioptas, A. Clauset, D. Kempe, and C. Moore, "On the bias of traceroute sampling: or, power-law degree distributions in regular graphs," in Proceedings of the thirty-seventh annual ACM symposium on Theory of computing (STOC). ACM, 2005, pp. 694-703.

[4] J. Guillaume and M. Latapy, "Relevance of massively distributed explorations of the Internet topology: Simulation results," in INFOCOM 2005. 24th Annual Joint Conference of the IEEE Computer and Communications Societies. Proceedings IEEE, vol. 2. IEEE, 2005, pp. 1084-1094.

[5] M. Latapy and C. Magnien, "Complex network measurements: Estimating the relevance of observed properties," in INFOCOM 2008. The 27th Conference on Computer Communications. IEEE. IEEE, 2008, pp. $1660-1668$

[6] D. Stutzbach, R. Rejaie, N. Duffield, S. Sen, and W. Willinger, "Sampling techniques for large, dynamic graphs," in INFOCOM 2006. 25th IEEE International Conference on Computer Communications. Proceedings. IEEE, 2006, pp. 1-6.

[7] - "On unbiased sampling for unstructured peer-to-peer networks," IEEE/ACM Transactions on Networking (TON), vol. 17, no. 2, pp. 377390, 2009.
[8] R. Albert, H. Jeong, and A. Barabasi, "Error and attack tolerance of complex networks," Nature, vol. 406, no. 6794, pp. 378-382, 2000.

[9] D. Callaway, M. Newman, S. Strogatz, and D. Watts, "Network robustness and fragility: Percolation on random graphs," Physical Review Letters, vol. 85, no. 25, pp. 5468-5471, 2000.

[10] R. Cohen, K. Erez, D. Ben-Avraham, and S. Havlin, "Resilience of the Internet to random breakdowns," Physical Review Letters, vol. 85, no. 21 , pp. 4626-4628, 2000.

[11] —, "Breakdown of the Internet under intentional attack," Physical Review Letters, vol. 86, no. 16, pp. 3682-3685, 2001.

[12] M. Faloutsos, P. Faloutsos, and C. Faloutsos, "On power-law relationships of the Internet topology," in ACM SIGCOMM Computer Communication Review, vol. 29, no. 4. ACM, 1999, pp. 251-262.

[13] L. Dall'Asta, I. Alvarez-Hamelin, A. Barrat, A. Vázquez, and A. Vespignani, "A statistical approach to the traceroute-like exploration of networks: theory and simulations," Arxiv preprint cond-mat/0406404, 2004.

[14] "Caida - skitter project," http://www.caida.org/tools/measurement/skitter/.

[15] Y. Shavitt and E. Shir, "DIMES: Let the Internet measure itself," ACM SIGCOMM Computer Communication Review, vol. 35, no. 5, pp. 71 74, October 2005.

[16] J. Pansiot and D. Grad, "On routes and multicast trees in the Internet," ACM SIGCOMM Computer Communication Review, vol. 28, no. 1, pp. 41-50, 1998 .

[17] C. Crespelle and F. Tarissan, "Evaluation of a new method for measuring the Internet degree distribution: Simulation results," Computer Communications, 2010.

[18] M. Latapy, E. Rotenberg, C. Crespelle, and F. Tarissan, "Measuring the Degree Distribution of Routers in the Core Internet," in IFIP Networking, 2014, to appear. 